\title{
Capabilities of Mdct of the Temporal Bone Structures Before Stapes Surgery
}

\author{
Bodrova I. V.', Mukhamatullina E. Z.', \\ Ternovoy S. K.', Lopatin A. S. ${ }^{2}$ \\ I I. M. Sechenov First Moscow State Medical University \\ 2 Federal state budgetary institution „Clinical Hospital № 7 " of \\ Administration of the President of the Russian Federation
}

\begin{abstract}
Introduction: The overall results of stapes surgery considerably depend on the anatomy peculiarities of complicated temporal bone's structure.

Materials and methods: 37 persons (63 ears) with otosclerosis participated in the study. All patients have been examined by microotoscopy, audiologic tests, MDCT. The niche of vestibular window was assessed in all ears on the following criteria: the width and form of the niche, the presence and absence of overhanging of facial nerve canal and promontorium over the vestibular window, the width of the stapes footplate, stapes cruses width, distance to the internal wall of vestibule. All 63 ears underwent stapes surgery.

Results: Overhanging of facial nerve canal over the vestibular window were observed in 14 ears, overhanging of a promontorium - in 9 ears, protrusion of facial nerve canal - in 6 ears. Wide niche was observed in 45 ears, narrow-in 18. The rectangular shape was observed in 18 ears, trapezoid-in 32, triangular-in 13. Thickening of a stapes footplate observed in all 53 ears. Thickening of stapes cruses noted in 11 ears. Distance to the internal wall of a vestibule less than $2 \mathrm{Mm}$ noted in 5 ears, more than 2 мм-in 58 . The stapedoplasty was performed in 63 ears: 44 the ,standard“ piston stapedoplasty; 14 the ,aim“ piston stapedoplasty; 5 the „standard“ piston stapedoplasty with stapedectomy. Sensitivity of MDCT was $95,2 \%$, specificity$98,4 \%$, accuracy $-96,8 \%$.

Conclusion: The MDCT-criteria allows estimating the complexity of the surgery, to plan surgery thoroughly, to predict the outcome.
\end{abstract}

Keywords: multidetector computed tomography, MDCT, otosclerosis, stapedoplasty

\section{Introduction}

To achieve a good functional result at the preoperative stage, it is important to evaluate in detail the anatomy and topography of the middle ear structures in relation to the niche of vestibular window, which is especially important for predicting the real outcome of the operation and the possibilities for its implementation for the specific surgeon [1]. The closer the anatomical interrelations of the middle ear structures in the vestibular window area, the worse the overview of the area of impact, the more technically difficult the intervention, the more likely the complications and the more important the need for quality visualization diagnostics $[2,3,4]$. Monitoring of anatomical and topographic features of the area of effect (the vestibular window) before the stapes surgery has not been carried out until recently. Ukkola-Pons E. et al. (2013) measured the height of the niche of vestibular window at one point along the coronal sections with the help of CT. The authors consider the niche of vestibular window of the threshold of $1.4 \mathrm{~mm}$ height and more - normal, $1.3 \mathrm{~mm}$ and less - narrow, which increases the risk of stapedoplasty [5]. Evaluation of the anatomical and topographic features of the vestibular window area before the stapes surgery is essential for prognostication stapedoplasty and requires the development of the above criteria. 


\section{Materials and methods}

37 persons (63 ears) with otosclerosis participated in the study. All patients were examined by microotoscopy, audiologic tests, MDCT. The CT study was performed using a bone filter with slice thickness of $0.5-0,625 \mathrm{~mm}$ in axial plane followed by multiplanar reconstructions.

We developed the MDCT- protocol to examine temporal bones before the stapes surgery for the first time (patent of the Russian Federation for invention No. 2491879 „Method for the prognostication of stapedoplasty"), which was applied in $100 \%$ of observations. The protocol included the following criteria for assessing the area of the forthcoming effect:

1) the presence (degree) of overhang and the integrity of facial nerve canal over the vestibular window;

2) the presence (degree) of overhang promontorium over the vestibular window;
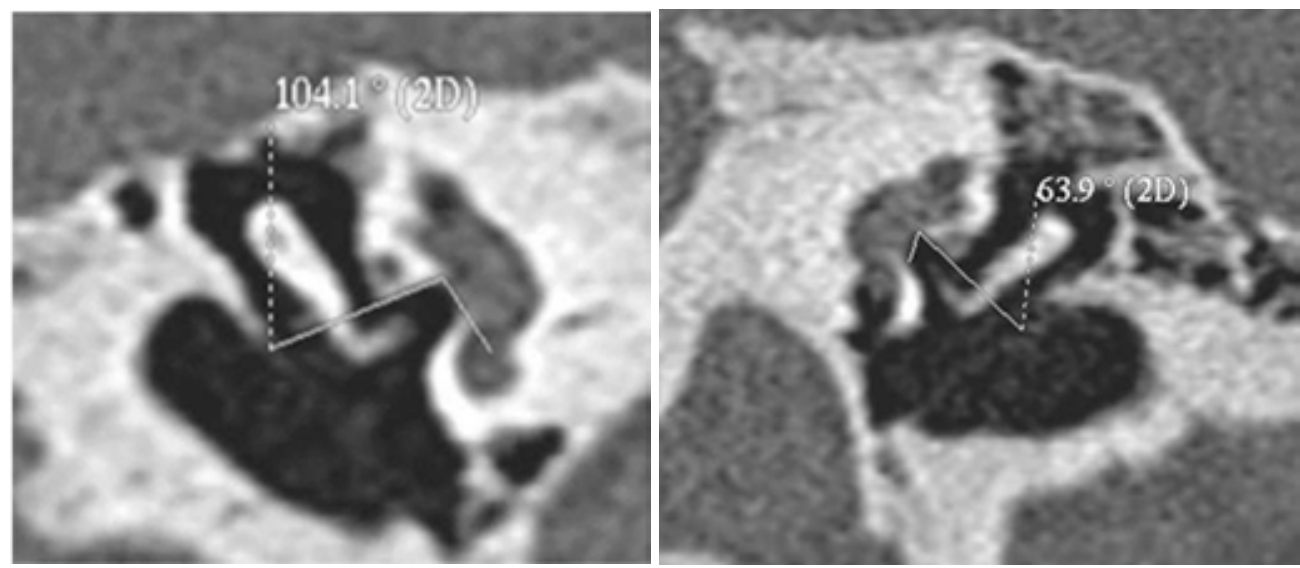

Fig. 1. The presence and extent of overhang of facial nerve canal over the vestibular window. A - absence of overhanging of facial nerve canal, B - presence of overhanging of facial nerve canal.
3) the width and form of the niche;

4) the thickness of the stapes footplate and stapes cruses;

5) the distance (critical) from the stapes footplate to the internal wall of vestibule.

The data obtained at MDCT was compared with the stapedoplasty protocols, which was performed in all patients (63 temporal bones).

\section{Results}

The presence (stagelgrade) of overhang of facial nerve canal over the vestibular window was estimated in an oblique projection by measuring the angle between the stapes footplate and the upper wall of the niche of vestibular window. At a value of $\geq 90^{\circ}$ it was considered that there were no overhangs -49 observations (78\%). At a value of $\leq 90^{\circ}$, it was considered that there is an overhang -14 observations (22\%) (Fig. 1).

The integrity of facial nerve canal was estimated on the basis of the absence of defects in the bone wall. The presence of a defect up to $6 \mathrm{~mm}$ in the bone wall of the canal (protrusion) was found in 6 observations (9\%) (Fig. 2).

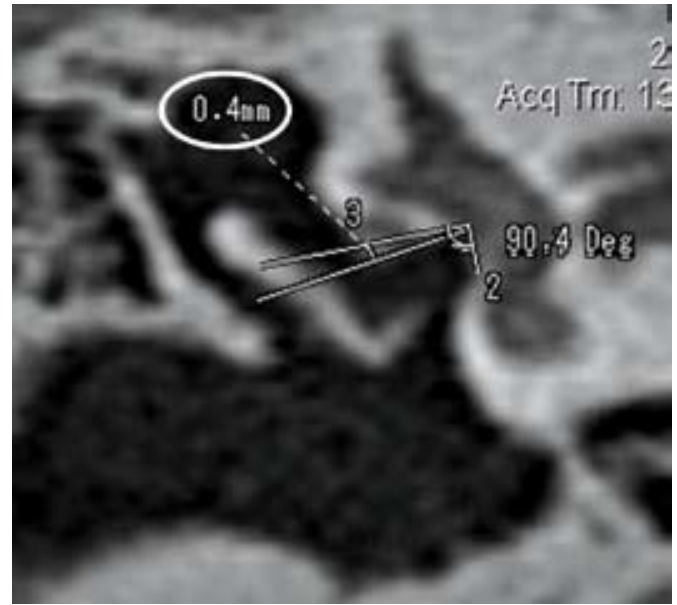

Fig. 2. Integrity of the canal of the facial nerve. Protrusion of facial nerve canal 0,4 мм. 
The presence (stagelgrade) of overhang promontorium over the vestibular window was estimated in an oblique projection by measuring the angle between the stapes footplate and the bottom wall of the niche of vestibular window. At a value of $\geq 90$ $\circ$ it was considered that there were no overhangs in 54 observations $(86 \%)$ ). At a value of $\leq 90^{\circ}$, it was considered that there is an overhang in 9 observations (14\%) (Fig. 3).

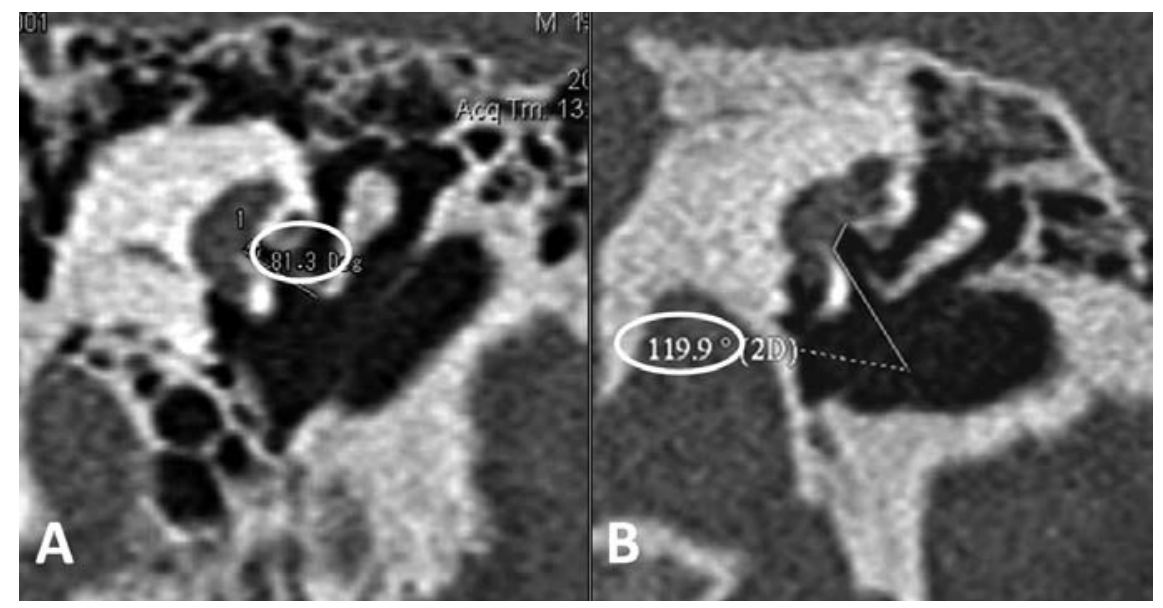

Fig. 3. The presence and extent of overhang of promontorium over the vestibular window. $\mathrm{A}$ - presence of promontorium overhanging, $\mathrm{B}$ - absence of promontorium overhanging.

Measurements of the width of the niche of vestibular window were made in an oblique projection using three measurements (Fig. 4):

1) the line was drawn along the stapes footplate;

2) the line was drawn in the middle of the niche of vestibular window and strictly parallel to the 1st line;
3) the line drawn on the ,exit“ of the niche of vestibular window and strictly parallel to the $1 \mathrm{st}$ and 2nd lines.
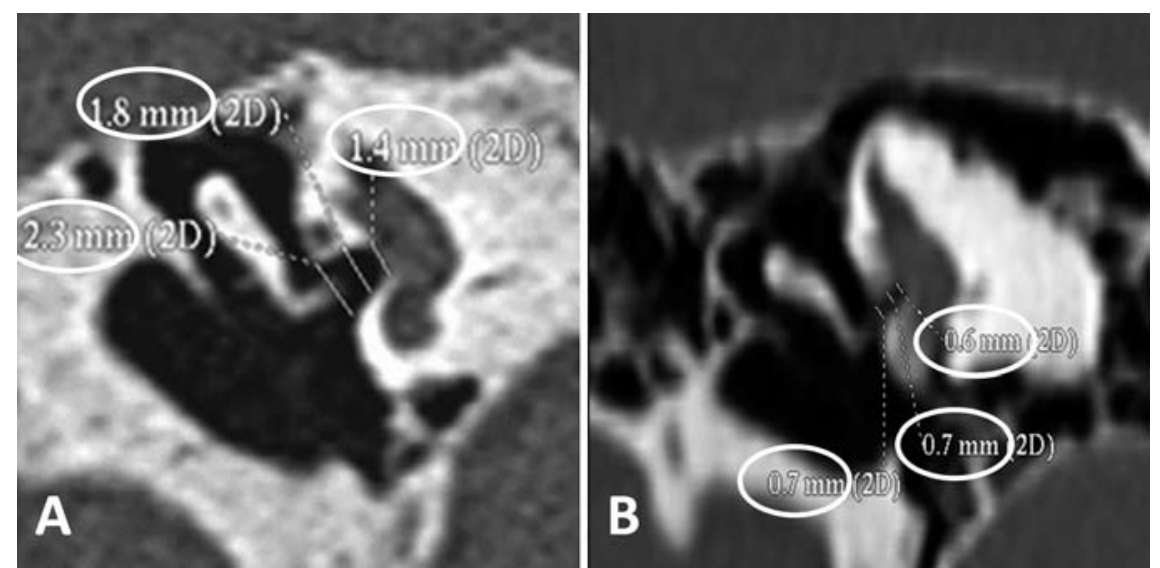

Fig. 4. Measurement of the width of the niche.

A - niche of vestibular window is wide, $B-$ niche of vestibular window is narrow.

At a value of niche $\geq 1 \mathrm{~mm}, 1.5 \mathrm{~mm}$ and $1.9 \mathrm{~mm}$ measured accordingly the lines it were counted as a wide niche of vestibular window in 45 observations (71\%). At a value of $\leq 1 \mathrm{~mm}, 1.5 \mathrm{~mm}$ and $1.9 \mathrm{~mm}$ measured accordingly the lines it were counted as a narrow niche of vestibular window in 18 observations $(29 \%)$.
The form of the niche of vestibular window was formed by summation of the data obtained above: the presence of the overhang of the facial nerve canal and the promontorial wall, as well as the width of the niche. The rectangular shape was observed in 18 cases $(29 \%)$, trapezoid in 32 observations $(51 \%)$, triangular in 13 observations (20\%) (Fig. 5). 

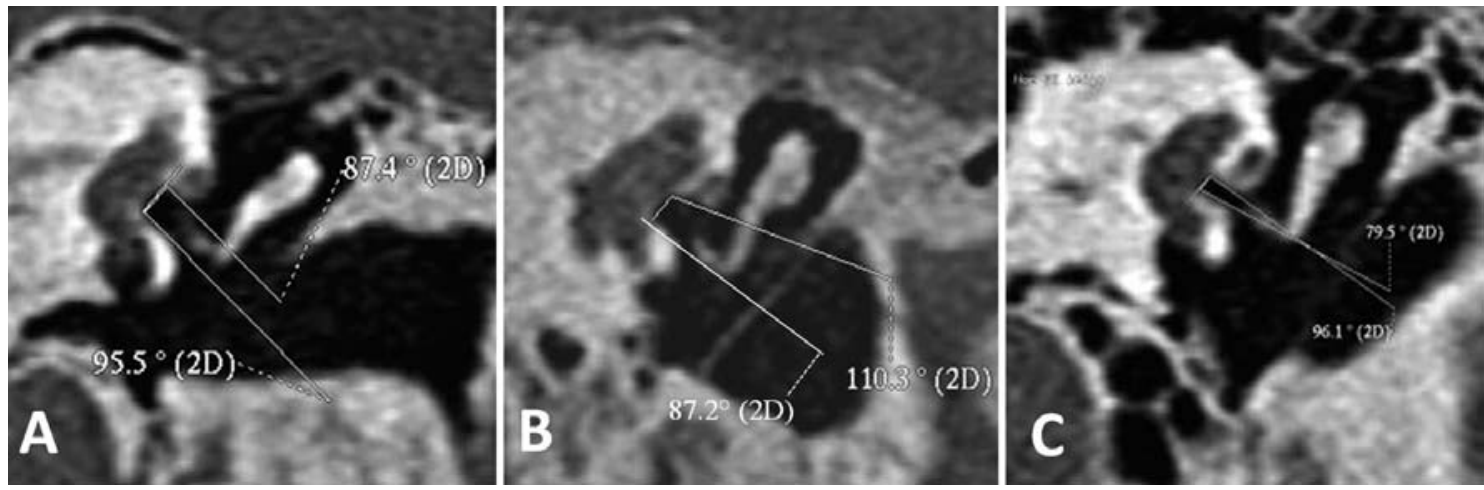

Fig. 5. The form of vestibular window niche.

$\mathrm{A}$ - the rectangular shape, $\mathrm{B}$ - the trapezoid shape, $\mathrm{C}$ - the triangular shape.

The thickness of the stapes footplate were measured in an axial projection using three measurements (Fig. 6):

1) the measurement in the front part of the footplate, at the level of attachment of the front stapes cruse.

2) the measurement in the middle part of the footplate.

3) the measurement at the back of the footplate, at the level of attachment of the back stapes cruse.

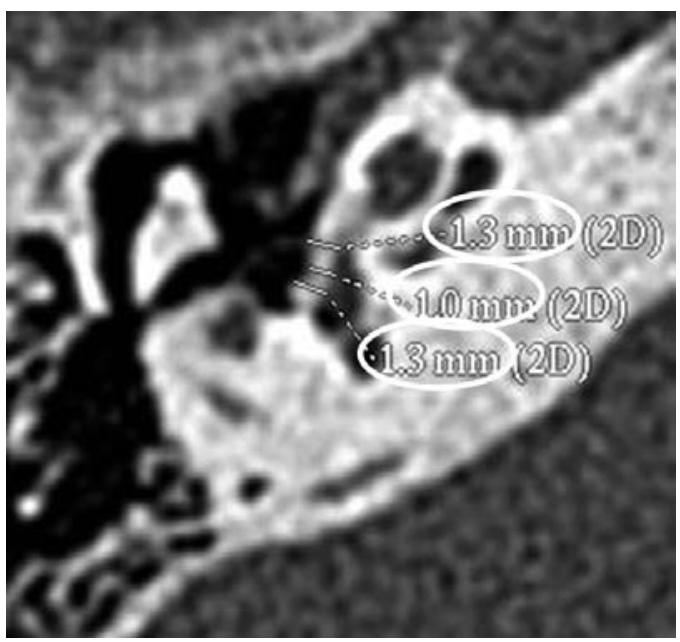

Fig. 6. Measurement of the thickness of the stapes footplate at three points. The maximum thickening of stapes footplate up $1,3 \mathrm{~mm}$.

At a value of $\leq 0.8 \mathrm{~mm}, 0.9 \mathrm{~mm}$ and $1 \mathrm{~mm}$ measured respectively the lines were counted that the thickness of the stapes footplate did not create additional difficulties in 32 observations (51\%). At a value of $\geq 0.8 \mathrm{~mm}, 0.9 \mathrm{~mm}$ and $1 \mathrm{~mm}$, measured respectively the lines were counted that the stapes footplate was thickened and would create additional difficulties accordingly in 31 observations (49\%).
The thickness of the stapes cruses were measured in an axial scans in their middle part. At a thickness value of $\leq 0.6 \mathrm{~mm}$, it were counted that the cruses were not thickened in 52 observations (83\%). At a thickness of $\geq 0.6 \mathrm{~mm}$, the cruses were counted as thickened in 11 observations (17\%) (Fig. 7).

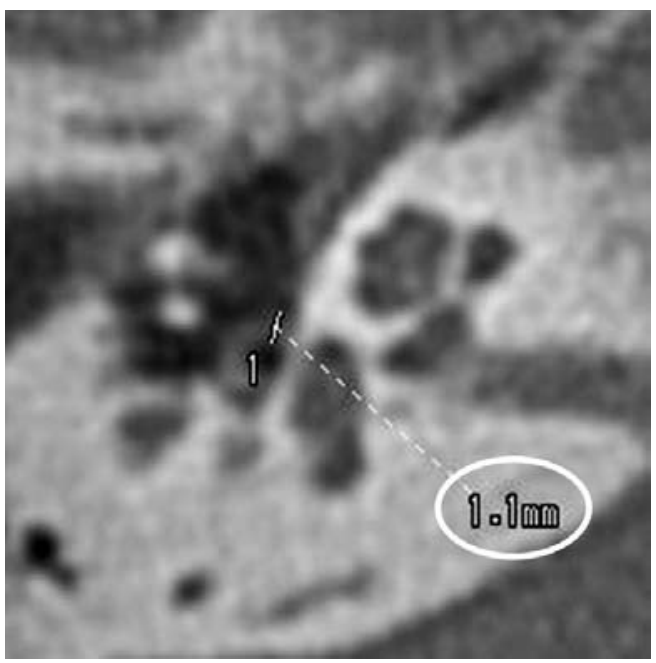

Fig. 7. Measurement of the thickness of the stapes cruses. The anterior stapes of the cruses is thickened up 1,1 $\mathrm{mm}$.

The critical distance to the internal wall of vestibule was measured in an oblique projection along the line, which was perpendicular to the stapes footplate and was drawn from its inner surface to the inner wall of the vestibule. At a distance $\geq 2 \mathrm{~mm}$, no difficulties were assumed (58 observations). At a distance of $\leq 2 \mathrm{~mm}$, special caution was required to conduct the platinotomy or stapedectomy ( 5 observations) (Fig. 8). 

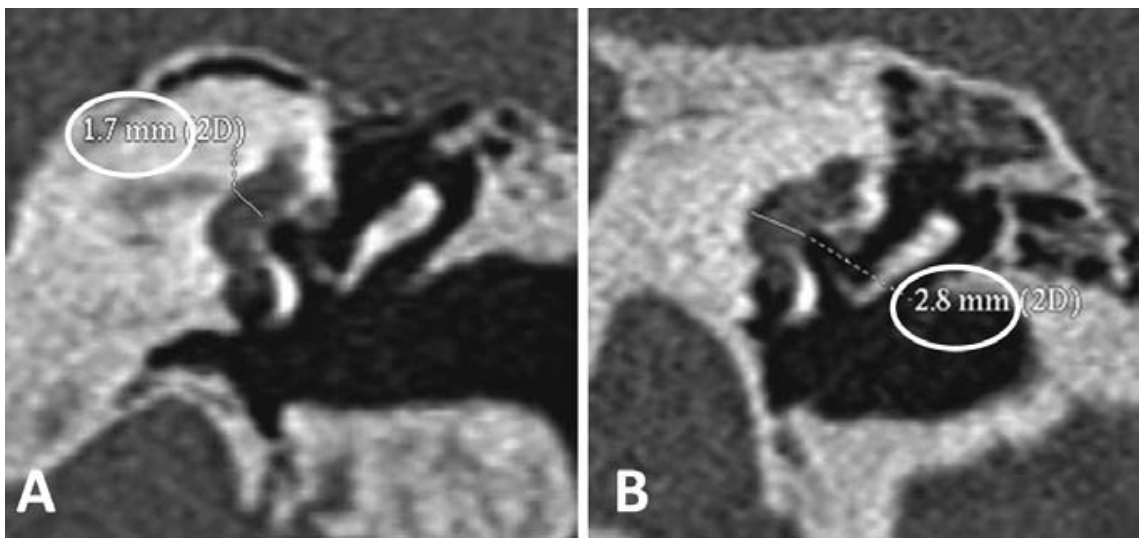

Fig. 8. The critical distance to the internal wall of vestibule.

A - the critical distance is less than $2 \mathrm{~mm}, \mathrm{~B}$ - the critical distance greater than $2 \mathrm{~mm}$.

In all 63 cases, stapedoplasty was performed along the preliminary protocol. In 44 observed patients, in whom the niche of vestibular window was wide, overhanging the facial nerve canal and the promontory wall above the niche of vestibular window was not noted, a „standard“ piston stapedoplasty was performed with the titanium prosthesis „K-Piston“ of the German firm „Kurz“.

In 14 cases, in which there was an overhang of the facial nerve canal above the niche of vestibular window, an ,aiming“ piston stapedoplasty was planned, that is, platinototomy was carried out in that part of the base of the stapes that was at the maximum distance from the overhanging facial nerve. The corresponding instrumentation was prepared for the operation (additional micro-hooks, diamond cutters, etc.) and it was provided two variant of implants: a conventional titanium prosthesis „piston“ (12 observations) and ,angular piston“ (2 observations). The MDCT data completely coincided with the intraoperative findings.

In five cases in which considerable technical difficulties created a massive focus of otospongosis, which was obturating niche of vestibular window, as well as the overhanging the facial nerve canal and the promontorium, as a result of which the niche of the vestibular window was extremely narrow. Therefore, the transcanal access were selected, stapedoplasty with a titanium prosthesis ,piston“ with the complete removal of focus of otospongosis and the stapes footplate (stapedectomy).

Based on the above, we have developed a table for the definition of the complexity category of stapedoplasty (Table 1), with which it is easier for an otosurgeologist to determine the stage of complexity of stapedoplasty and the risk of intraoperative complications.

Table 1. The table of definition of the complexity category of stapedoplasty

\begin{tabular}{|c|c|c|c|}
\hline $\begin{array}{c}\text { Category } \\
\text { MDCT-criteria }\end{array}$ & $\begin{array}{c}0 \\
\text { There is } \\
\text { no risk }\end{array}$ & $\begin{array}{c}1 \\
\text { The risk is present }\end{array}$ & $\begin{array}{c}2 \\
\text { The risk is high }\end{array}$ \\
\hline The wall of the facial nerve canal & $\geq 90 \epsilon$ & from $80 \epsilon$ to $90 \epsilon$ & $\leq 80 €$ \\
\hline The wall of the promontorium & $\geq 90 \epsilon$ & from $80 €$ to $90 €$ & $\leq 80 \epsilon$ \\
\hline The width of the niche of vestibular window & $\geq 0,5 \mathrm{~mm}$ & $0-0,5 \mathrm{~mm}$ & $\leq 0 \mathrm{~mm}$ \\
\hline The form of the niche of vestibular window & trapezoid, rectangular & $\begin{array}{l}\text { rectangular, } \\
\text { trapezoid }\end{array}$ & triangular \\
\hline The maximum thickness of the stapes footplate & $\leq 1$ & От 1 до $1,5 \mathrm{~mm}$ & $\geq 1,5 \mathrm{~mm}$ \\
\hline The maximum thickness of the stapes cruses & $\leq 0,6 \mathrm{~mm}$ & $\geq 0,6 \mathrm{~mm}$ & $\geq 1 \mathrm{~mm}$ \\
\hline $\begin{array}{l}\text { The critical distance to the internal wall of } \\
\text { vestibule }\end{array}$ & $\geq 2 \mathrm{~mm}$ & $\leq 2 \mathrm{~mm}$ & $\leq 1,5 \mathrm{~mm}$ \\
\hline The presence of protrusions of the facial nerve & - & + & + \\
\hline
\end{tabular}


If all the indicators fall into the „, 0 " category, then we believe that there is no risk. If at least one of the indicators falls into the graph with the category „1" - there is a risk. If one of the indicators falls into the graph with the category ,2“ - the risk is particularly high.

The sensitivity of the MDCT of the temporal bones according to the developed protocol was $95.24 \%$, specificity $98.4 \%$, accuracy $96.79 \%$. MDCT data coincided with intraoperative findings in 60 observations, which can be explained by the development of the optimal MDCT protocol at the stage of its development.

\section{Discussions}

Ukkola-Pons E. et al. (2013) measured the height of the niche of vestibular window at one point along the coronal sections with the help of CT. The authors consider the niche of vestibular window of the threshold of $1.4 \mathrm{~mm}$ height and more - normal, $1.3 \mathrm{~mm}$ and less - narrow, which increases the risk of stapedoplasty [5]. Cavaliere M. et al. (2012) in their studies did not reveal a correlation between the diameter of the stapes prosthesis, but the authors recommend to install a prosthesis smaller diameter with a narrow niche of the vestibule and overhanging of the facial nerve [6].

In the described publications, only the width of the niche of vestibular window before stapedoplasty was evaluated, while all the anatomical structures of the niche of vestibular window area play an important role in the successful stapedoplasty.
According that the niche of vestibular window were estimated with the following parameters: the wall of the facial nerve canal (overhang presents/ no), the promontorial wall (overhang presents/ no), the width of the niche of vestibular window (the difference between the proximal and distal height), the shape of the niche of vestibular window, the maximum thickness of the stapes footplate and stapes cruses, the critical distance to the internal wall of vestibule, the presence of protrusions of the facial nerve.

The developed MDCT protocol of temporal bones before the stapes surgery (patent of the Russian Federation for invention No. 2491879 „Method for the prediction of stapedoplasty") allowed optimally plan the forthcoming surgical intervention on the stapes and obtain a good functional result, and the developed table for determining the complexity category stapedoplasty allowed to avoid intraoperative complications. High sensitivity (95.24\%) and specificity $(98.4 \%)$ of the preoperative way of predicting stapedoplasty were proved.

\section{Conclusion}

The developed MDCT protocol of temporal bones before the stapes surgery allowed to determine the correct surgical tactics at the preoperative stage, to conduct thorough preparation for the operation and, as a result, to obtain a good functional result, and to avoid frequent intraoperative complications in such cases.

\title{
References:
}

1. Hildmann H., Sudhoff H. Middle Ear Surgery. — Berlin, Germany: Springer-Verlag, New York: Heidelberg; 2006. — P. 112-119.

2. Perez-Lazaro J. Effectiveness assessment of otosclerosis surgery // Acta Oto-Laryng. — 2005. — Vol. 125 (3). — P. $935-945$.

3. Waleed R.A., Ramez R.B., Mohamed A.M. Significance of high resolution CT scan in otosclerosis // Med. J. Cairo Univ. — 2011. — Vol. 79. - P. 7-12.

4. Wiet R.J. Ear and temporal bone surgery: minimizing risks and complications. - New York-Stuttgart: Theim, 2005 . -320 p.

5. Ukkola-Pons E., Ayache D., Pons Y., Ratajczak M., Nioche C., Williams M. Oval window niche height: quantitative evaluation with CT before stapes surgery for otosclerosis // AJNR Am J Neuroradiol. — 2013. - Vol. 34 (5). - P. 1082-1085.

6. Cavaliere M., Ricciardiello F., Mesolella M., Iengo M. Stapedotomy: functional results with different diameter prostheses // ORL J Otorhinolaryngol Relat Spec. — 2012. — Vol. 74 (2). — P. 93-96.

\author{
Bodrova Irina \\ 121614 , \\ Russia, Moscow, \\ Osennyaya str., h.22, ap.176 \\ $+79037957417$ \\ $+74957957417$ \\ iv-bodrova@mail.ru
}

